Department of Engineering Mechanics, and International Research Center for

Computational Mechanics,

Dalian University of Technology, Dalian 116024, China

State Key Laboratory of Digital Manufacturing Equipment and Technology, Huazhong University of Science and Technology, Wuhan 430074, China e-mail: ruili@dlut.edu.cn

Pengcheng Wang

State Key Laboratory of Structural Analysis for Industrial Equipment,

Department of Engineering Mechanics, and International Research Center for

Computational Mechanics,

Dalian University of Technology, Dalian 116024, China

Bo Wang

College of Engineering, Peking University,

Beijing 100871, China

Chunyu Zhao

CAS Key Laboratory of Mechanical Behavior and Design of Materials,

Department of Modern Mechanics, University of Science and Technology of China, Hefei 230027, China

Yewang $\mathrm{Su}^{1}$

State Key Laboratory of Nonlinear Mechanics, Institute of Mechanics,

Chinese Academy of Sciences, Beijing 100190, China;

School of Engineering Science, University of Chinese Academy of Sciences, Beijing 100049, China e-mail: yewangsu@imech.ac.cn

\section{New Analytic Free Vibration Solutions of Rectangular Thick Plates With a Free Corner by the Symplectic Superposition Method}

Seeking analytic free vibration solutions of rectangular thick plates without two parallel simply supported edges is of significance for an insight into the performances of related engineering devices and structures as well as their rapid design. A challenging set of problems concern the vibrating plates with a free corner, i.e., those with two adjacent edges free and the other two edges clamped or simply supported or one of them clamped and the other one simply supported. The main difficulty in solving one of such problems is to find a solution meeting both the boundary conditions at each edge and the condition at the free corner, which is unattainable using a conventional analytic method. In this paper, for the first time, we extend a novel symplectic superposition method to free vibration of rectangular thick plates with a free corner. The analytic frequency and mode shape solutions are both obtained and presented via comprehensive numerical and graphic results. The rigorousness in mathematical derivation and rationality of the method (without any predetermination for the solutions) guarantee the validity of our analytic solutions, which themselves are also validated by the reported results and refined finite element analysis. [DOI: 10.1115/1.4038951]

Keywords: thick plate, free corner, free vibration, analytic solution, symplectic superposition method

\section{Introduction}

Solving the free vibration problems of plate structures has been an important topic in mechanical engineering, which is very useful for the performance analysis and design of related engineered devices and structures. Although many progresses have been gained in seeking the solutions of plates with various shapes, as summarized in Leissa's seminal monograph [1], analytic solutions are far from complete due to the mathematical challenge in treating the plate models such as the Kirchhoff thin plate and more complex Mindlin/Reissner thick plate [2,3] or the others [4,5]. Conventional approximate/numerical methods include the finite element method (FEM), finite difference method, Rayleigh-Ritz method, Galerkin method, series method, etc. The reader is

\footnotetext{
${ }^{1}$ Corresponding authors.

Contributed by the Technical Committee on Vibration and Sound of ASME for publication in the Journal of Vibration and Acoustics. Manuscript received September 10, 2017; final manuscript received January 4, 2018; published online February 9, 2018. Assoc. Editor: Izhak Bucher.
}

referred to Leissa's work [1] for more details, where comprehensive results are available for vibration of thin plates.

Many recently developed novel numerical methods have been explored for plates' vibration problems. Some representative methods are briefly reviewed in the following. Wu and $\mathrm{Zhu}$ [6] developed a new global spatial discretization method to accurately calculate natural frequencies and dynamic responses of twodimensional continuous systems such as membranes and thin plates, where much fewer degrees-of-freedom and much less computational effort are needed compared with the FEM and finite difference method. Wang [7] presented the frequencies and mode shapes for the rounded rectangular thin plate with completely free edges by using a family of homotopy shapes with low powers, which greatly facilitate differentiation and numerical integrations for an efficient Ritz method. Leamy [8] proposed a phase closure approach for finding semi-exact, closed-form expressions for the natural frequencies of thin rectangular plates; the approach has the advantage of yielding highly accurate, closed-form algebraic expressions suitable for archiving in compilations such as 
handbooks and manuals. Waksmanski et al. [9] obtained an exact closed-form solution for free vibration of a simply supported and multilayered one-dimensional quasi-crystal plate by using the pseudo-Stroh formulation and propagator matrix method; the work is very useful for further expanding the applications of quasi-crystal, especially when used for composite materials. Lai and Xiang [10] applied the discrete singular convolution method to investigate the buckling and vibration of heavy standing thin plates and obtained accurate first-known vibration solutions for elastically restrained vertical plates subjecting to body forces/selfweight. Besides, Malekzadeh and Karami [11] presented a differential quadrature procedure for free vibration analysis of moderately thick plates with variable thickness on two-parameter elastic foundations under in-plane edge forces; accurate results were obtained for higher-order modes of vibration with only few grid points, which demonstrates the advantage of low computation cost of the method. Cho et al. [12] applied the assumed mode method and mode superposition method to develop a very simple, fast, and accurate procedure for the forced vibration analysis of plates and stiffened panels, which is especially appropriate for early design stage when different dimensions of plates and stiffened panels and their influence on dynamic response are examined. In addition to these, the Rayleigh-Ritz method is still popular as a very efficient way to handle plates' vibration problems. The updates on the method focus on the selection of some novel admissible functions, yielding different expressions of displacement components. For example, Zhou et al. [13] utilized the admissible functions comprising the Chebyshev polynomials multiplied by a boundary function for free vibration analysis of rectangular plates with any thicknesses. Pradhan et al. [14] expressed the trial functions as the linear combinations of simple algebraic polynomials to study free vibration of thick rectangular plates based on new inverse trigonometric shear deformation theories. Ye et al. [15] adopted a new form of trigonometric series expansion to develop a modified Fourier solution for the free vibration problems of moderately thick rectangular plates based on the firstorder shear deformation theory. Similar treatments were applied by Jin et al. [16] to obtain three-dimensional solution for the free vibrations of arbitrarily thick functionally graded rectangular plates, and by Zhang et al. [17] for the free vibration analysis of moderately thick rectangular plates with non-uniform boundary conditions.

In comparison with the numerous numerical methods, analytic approaches to plates' vibration problems are much fewer. For rectangular plates, it is known that the conventional semi-inverse Lévy method is only applicable to those having at least two parallel simply supported edges. Although some successful attempts have been made in recent years on obtaining the analytic solutions of intractable rectangular thin plate problems, including bending, vibration, and buckling of plates without two parallel simply supported edges [18-20], there have been rare reports for analytic vibration solutions of thick plates. Few available achievements are found for rectangular thick plates with only clamped and/or simply supported edges, as reported by Xing and Liu [21,22], or for plates with only one free edge, as reported by Li et al. [23]. The free vibration of rectangular thick plates with a free corner, i.e., with two adjacent edges free and the other two edges clamped or simply supported or one of them clamped and the other one simply supported, have not been solved analytically. The problems of this type are very interesting because not only the free edges but also the free corner is involved, whose analytic solutions are, however, very difficult to acquire due to the predicament in finding a solution meeting both the boundary conditions at each edge and the condition at the free corner. Therefore, seeking a novel analytic method becomes indispensable to settle these problems.

In recent years, a symplectic elasticity approach has been proposed by Yao et al. [24]. Compared with the classic analytic approaches such as the semi-inverse method, the symplectic approach is conducted in the Hamiltonian system, in physics, and in the symplectic space, in mathematics. Some useful techniques, such as the separation of variables that is sometimes invalid in the Euclid space, become valid in the symplectic space, which makes it possible to explore newer analytic solutions. The approach has been extended to many fields [25], among which the plate problems were further advanced by Lim et al. [26,27] and Li et al. $[18,19,23,28]$. In this paper, we obtain the analytic free vibration solutions of rectangular thick plates with a free corner by an elegant symplectic superposition method, which combines the rationality of the symplectic elasticity approach and generality of the superposition method such that the focused problems could be solved in a rigorous way, without any trial solutions predetermined. In the following, we first construct a fundamental free vibration problem, i.e., a vibrating plate with two adjacent edges slidingly clamped and the other two edges simply supported. With the Hamiltonian system-based variable separation in the symplectic space, which triggers an eigenvalue problem, and the symplectic eigen expansion, the analytic solution of the fundamental problem is obtained. By superposition of two fundamental solutions followed by its equivalence with the original problem, the equation for determining the natural frequencies is achieved. The mode shape associated with a frequency solution is then readily obtained. Comprehensive numerical and graphic results are provided for the plates under consideration, which offer the benchmarks for various emerging solution methods.

\section{Fundamental Free Vibration Problem With Solution in the Symplectic Space}

In a recently published study [23], we have constructed the governing equations of a Mindlin theory-based thick plate's free vibration in the symplectic space in the rectangular coordinate system xoy (Fig. 1(a)), which yields a Hamiltonian system-based matrix-form expression, written by

$$
\partial \mathbf{Z} / \partial y=\mathbf{H Z}
$$

where

$$
\mathbf{Z}=[\Phi, w, \Psi, \alpha, \beta, \theta]^{\mathrm{T}}
$$

is the state vector, incorporating the modal displacement $w$ and five other functions, i.e., $\Phi, \Psi, \alpha, \beta$, and $\theta$, defined by

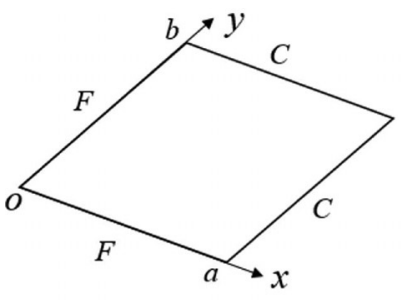

(a)

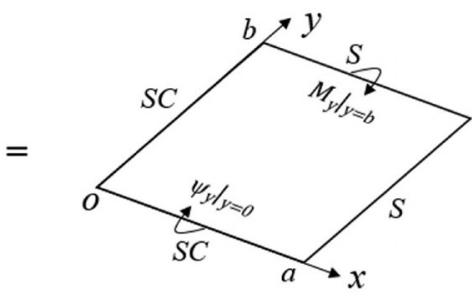

(b)

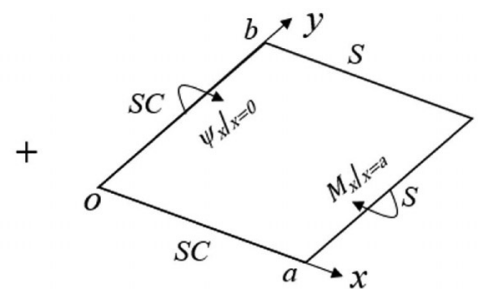

$(c)$

Fig. 1 Symplectic superposition for free vibration of a CCFF thick plate 


$$
\begin{gathered}
\Phi=\frac{\partial \psi_{x}}{\partial x}+\frac{\partial \psi_{y}}{\partial y} \\
\Psi=\frac{\partial \psi_{y}}{\partial x}-\frac{\partial \psi_{x}}{\partial y} \\
\alpha=\frac{D}{\rho h \omega^{2}} \frac{\partial \Phi}{\partial y} \\
\beta=\frac{\partial w}{\partial y}
\end{gathered}
$$

and

$$
\theta=\frac{\partial \Psi}{\partial y}
$$

Here, $\psi_{x}$ and $\psi_{y}$ denote the rotating angles about the $y$ - and $x$ axes, respectively; $\rho$ is the mass density of plate, $h$ the thickness, $\omega$ the natural frequency, and $D$ the flexural stiffness. The Hamiltonian matrix $\mathbf{H}$ has the form

$$
\mathbf{H}=\left[\begin{array}{ll}
\mathbf{0} & \mathbf{F} \\
\mathbf{G} & \mathbf{0}
\end{array}\right]
$$

with

$$
\mathbf{F}=\left[\begin{array}{ccc}
\rho h \omega^{2} / D & 0 & 0 \\
0 & 1 & 0 \\
0 & 0 & 1
\end{array}\right]
$$

and

$$
\mathbf{G}=\left[\begin{array}{ccc}
-\frac{D}{\rho h \omega^{2}} \frac{\partial^{2}}{\partial x^{2}} & 1 & 0 \\
1 & -\frac{\partial^{2}}{\partial x^{2}}-\frac{\rho h \omega^{2}}{C} & 0 \\
0 & 0 & \frac{10}{h^{2}}-\frac{\partial^{2}}{\partial x^{2}}
\end{array}\right]
$$

where $C$ is the shear stiffness. If Eq. (1) is successfully solved, the key quantities for a thick plate's free vibration can be readily obtained via $w, \Phi$, and $\Psi$. For example, besides $w$, the other two general modal displacements $\psi_{x}$ and $\psi_{y}$ are obtained by

$$
\begin{aligned}
& \psi_{x}=\frac{\partial w}{\partial x}+\frac{D}{C}\left[\frac{\partial \Phi}{\partial x}-\frac{1-v}{2} \frac{\partial \Psi}{\partial y}\right] \\
& \psi_{y}=\frac{\partial w}{\partial y}+\frac{D}{C}\left[\frac{\partial \Phi}{\partial y}+\frac{1-v}{2} \frac{\partial \Psi}{\partial x}\right]
\end{aligned}
$$

Then all other quantities can be obtained, e.g., the bending moments $M_{x}=-D\left(\partial \psi_{x} / \partial x+\nu \partial \psi_{y} / \partial y\right)$ and $M_{y}=-D\left(\partial \psi_{y} / \partial y\right.$ $\left.+\nu \partial \psi_{x} / \partial x\right)$, twisting moment $M_{x y}=-D(1-\nu)\left(\partial \psi_{x} / \partial y\right.$ $\left.+\partial \psi_{y} / \partial x\right) / 2$, shear forces $Q_{x}=C\left(\partial w / \partial x-\psi_{x}\right)$ and $Q_{y}=C\left(\partial w / \partial y-\psi_{y}\right)$, with $\nu$ as the Poisson's ratio. Therefore, $w$, $\Phi$, and $\Psi$ are regarded as the governing functions in the present solution system. The main advantage of the description in the symplectic space via the Hamiltonian system-based governing equation (1) is the applicability of some powerful mathematical techniques such as the separation of variables and symplectic eigen expansion, which are not necessarily attainable in the Euclidean space [24]. As a result, seeking the analytic solutions to some complex problems becomes probable.
For free vibration of the plates with a free corner, constructing a fundamental problem is necessary in the symplectic superposition method. As shown in Fig. 1(b), we first investigate a plate slidingly clamped at $x=0$ and simply supported at $x=a$, with a rotating angle $\left.\psi_{y}\right|_{y=0}$ and a bending moment $\left.M_{y}\right|_{y=b}$ imposed at the slidingly clamped edge $y=0$ and the simply supported edge $y=b$, respectively. The boundary conditions of the plate are

$$
\begin{aligned}
\left.\psi_{x}\right|_{x=0}=0, & \left.Q_{x}\right|_{x=0}=0, & \left.M_{x y}\right|_{x=0}=0 \\
\left.w\right|_{x=a}=0, & \left.\psi_{y}\right|_{x=a}=0, & \left.M_{x}\right|_{x=a}=0
\end{aligned}
$$

and

$$
\begin{aligned}
\left.\psi_{y}\right|_{y=0} & =\sum_{m=1,3,5, \ldots}^{\infty} E_{m} \cos \left(\alpha_{m} x\right),\left.\quad Q_{y}\right|_{y=0}=0,\left.\quad M_{x y}\right|_{y=0}=0 \\
\left.w\right|_{y=b} & =0,\left.\quad \psi_{x}\right|_{y=b}=0,\left.\quad M_{y}\right|_{y=b}=\sum_{m=1,3,5, \ldots}^{\infty} F_{m} \cos \left(\alpha_{m} x\right)
\end{aligned}
$$

where $\left.\psi_{y}\right|_{y=0}$ and $\left.M_{y}\right|_{y=b}$ are expressed by the Fourier expansion, with $\alpha_{m}=m \pi /(2 a)$ and the constants $E_{m}$ and $F_{m}(m=1,3,5, \ldots)$ to be determined later. A similar fundamental problem is shown in Fig. 1(c), where the plate is slidingly clamped at $y=0$ and simply supported at $y=b$, with a rotating angle $\left.\psi_{x}\right|_{x=0}$ and a bending moment $\left.M_{x}\right|_{x=a}$ imposed at the slidingly clamped edge $x=0$ and the simply supported edge $x=a$, respectively. The boundary conditions are

$$
\begin{aligned}
\left.\psi_{y}\right|_{y=0}=0, & \left.Q_{y}\right|_{y=0}=0, & \left.M_{x y}\right|_{y=0}=0 \\
\left.w\right|_{y=b}=0, & \left.\psi_{x}\right|_{y=b}=0, & \left.M_{y}\right|_{y=b}=0
\end{aligned}
$$

and

$$
\begin{aligned}
\left.\psi_{x}\right|_{x=0} & =\sum_{m=1,3,5, \ldots}^{\infty} G_{m} \cos \left(\beta_{m} y\right),\left.\quad Q_{x}\right|_{x=0}=0,\left.\quad M_{x y}\right|_{x=0}=0 \\
\left.w\right|_{x=a} & =0,\left.\quad \psi_{y}\right|_{x=a}=0,\left.\quad M_{x}\right|_{x=a}=\sum_{m=1,3,5, \ldots}^{\infty} H_{m} \cos \left(\beta_{m} y\right)
\end{aligned}
$$

where $\beta_{m}=m \pi /(2 b), G_{m}$ and $H_{m}(m=1,3,5, \ldots)$ are the constants to be determined. It is found that the second problem (Fig. 1(c)) is actually the same as the first one (Fig. 1(b)) after the coordinates exchange. Therefore, only the first fundamental problem needs to be solved.

In the symplectic space, the separation of variables is valid [24], which implies that $\mathbf{Z}=\mathbf{X}(x) Y(y)$ can be applied to Eq. (1). yielding $\mathrm{d} Y(y) / \mathrm{d} y=\mu Y(y)$ and $\mathbf{H X}(x)=\mu \mathbf{X}(x)$, where $\mathbf{X}(x)=$ $[\Phi(x), w(x), \Psi(x), \alpha(x), \beta(x), \theta(x)]^{\mathrm{T}}$ is the eigenvector, with $\mu$ as the eigenvalue. Combining the boundary conditions expressed in Eq. (12), we derive the eigenvalues

$$
\begin{aligned}
& \mu_{ \pm m}^{(1)}= \pm \sqrt{\alpha_{m}^{2}-\bar{S} \bar{\omega}^{2} / a^{2}} \\
& \mu_{ \pm m}^{(2)}= \pm \sqrt{\alpha_{m}^{2}-\bar{R} \bar{\omega}^{2} / a^{2}} \\
& \mu_{ \pm m}^{(3)}= \pm \sqrt{\alpha_{m}^{2}+10 / h^{2}}
\end{aligned}
$$

and the associated eigenvectors 


$$
\begin{aligned}
& \mathbf{X}_{ \pm m}^{(1)}(x)=\left[\bar{R} \bar{\omega}^{2} \cos \left(\alpha_{m} x\right), a^{2} \cos \left(\alpha_{m} x\right), 0, \mu_{ \pm m}^{(1)} \bar{R} a^{4} \cos \left(\alpha_{m} x\right), \mu_{ \pm m}^{(1)} a^{2} \cos \left(\alpha_{m} x\right), 0\right]^{\mathrm{T}} \\
& \mathbf{X}_{ \pm m}^{(2)}(x)=\left[\bar{S} \bar{\omega}^{2} \cos \left(\alpha_{m} x\right), a^{2} \cos \left(\alpha_{m} x\right), 0, \mu_{ \pm m}^{(2)} \bar{S} a^{4} \cos \left(\alpha_{m} x\right), \mu_{ \pm m}^{(2)} a^{2} \cos \left(\alpha_{m} x\right), 0\right]^{\mathrm{T}} \\
& \mathbf{X}_{ \pm m}^{(3)}(x)=\left[0,0, \sin \left(\alpha_{m} x\right), 0,0, \mu_{ \pm m}^{(3)} \sin \left(\alpha_{m} x\right)\right]^{\mathrm{T}}
\end{aligned}
$$

for $m=1,3,5, \ldots$, where $\bar{\omega}=\omega a^{2} \sqrt{\rho h / D}, \bar{R}=\left(\delta-\sqrt{4 / \bar{\omega}^{2}+\delta^{2}}\right) / 2$, and $\bar{S}=\left(\delta+\sqrt{4 / \bar{\omega}^{2}+\delta^{2}}\right) / 2$, with $\delta=D /\left(\mathrm{Ca}^{2}\right)$. The solution of Eq. (1) is written as

$$
\mathbf{Z}=\sum_{m=1,3,5, \ldots}^{\infty}\left[f_{m}^{(1)} \mathrm{e}^{\mu_{m}^{(1)} y} \mathbf{X}_{m}^{(1)}(x)+f_{-m}^{(1)} \mathrm{e}^{-\mu_{m}^{(1)} y} \mathbf{X}_{-m}^{(1)}(x)+f_{m}^{(2)} \mathrm{e}^{\mu_{m}^{(2)} y} \mathbf{X}_{m}^{(2)}(x)+f_{-m}^{(2)} \mathrm{e}^{-\mu_{m}^{(2)} y} \mathbf{X}_{-m}^{(2)}(x)+f_{m}^{(3)} \mathrm{e}^{\mu_{m}^{(3)} y} \mathbf{X}_{m}^{(3)}(x)+f_{-m}^{(3)} \mathrm{e}^{-\mu_{m}^{(3)} y} \mathbf{X}_{-m}^{(3)}(x)\right]
$$

where $f_{ \pm m}^{(1)}$ to $f_{ \pm m}^{(3)}(m=1,3,5, \ldots)$ are the constant coefficients, determined by substitution of the required quantities obtained by $w, \Phi$, and $\Psi$ from Eq. (18) into Eq. (13). The resultant solutions of the governing functions for the first fundamental problem, denoted by $w_{1}$, $\Phi_{1}$, and $\Psi_{1}$, respectively, are

$$
\begin{aligned}
w_{1}= & a \times \sum_{m=1,3,5, \ldots}^{\infty} \frac{\cos (m \pi \bar{x} / 2)}{20 \delta \xi_{m} \eta_{m} \bar{\omega}^{2}(\bar{R}-\bar{S})} \times\left\{\phi\left\{\epsilon_{m} \eta_{m} \operatorname{sech} \xi_{m} \operatorname{sh}\left[\xi_{m}(1-\bar{y})\right]-\zeta_{m} \xi_{m} \operatorname{sech} \eta_{m} \operatorname{sh}\left[\eta_{m}(1-\bar{y})\right]\right\} E_{m}\right. \\
& \left.+20 \delta \xi_{m} \eta_{m}\left[\operatorname{sech} \xi_{m} \operatorname{ch}\left(\xi_{m} \bar{y}\right)-\operatorname{sech} \eta_{m} \operatorname{ch}\left(\eta_{m} \bar{y}\right)\right] \bar{F}_{m}\right\} \\
\Phi_{1}= & \frac{1}{a} \times \sum_{m=1,3,5, \ldots}^{\infty} \frac{\cos (m \pi \bar{x} / 2)}{20 \delta \xi_{m} \eta_{m}(\bar{R}-\bar{S})} \times\left\{\phi\left\{\bar{S} \epsilon_{m} \eta_{m} \operatorname{sech} \xi_{m} \operatorname{sh}\left[\xi_{m}(1-\bar{y})\right]-\bar{R} \xi_{m} \zeta_{m} \operatorname{sech} \eta_{m} \operatorname{sh}\left[\eta_{m}(1-\bar{y})\right]\right\} E_{m}\right. \\
& \left.+20 \delta \xi_{m} \eta_{m}(\bar{R}-\bar{S})\left[\bar{S} \operatorname{sech} \xi_{m} \operatorname{ch}\left(\xi_{m} \bar{y}\right)-\bar{R} \operatorname{sech} \eta_{m} \operatorname{ch}\left(\eta_{m} \bar{y}\right)\right] \bar{F}_{m}\right\}
\end{aligned}
$$

and

$$
\Psi_{1}=\frac{1}{a} \times \sum_{m=1,3,5, \ldots}^{\infty} \frac{m \pi \operatorname{sech} \gamma_{m} \sin (m \pi \bar{x} / 2)}{5 \delta \gamma_{m}(1-\nu)}\left\{5 \delta \phi \operatorname{sh}\left(\gamma_{m} \bar{y}\right) \bar{F}_{m}-\gamma_{m} \bar{h}^{2} \operatorname{ch}\left[\gamma_{m}(1-\bar{y})\right] E_{m}\right\}
$$

where $\bar{x}=x / a, \bar{y}=y / b, \quad \phi=b / a, \bar{F}_{m}=a F_{m} / D, \quad \xi_{m}=\phi \sqrt{m^{2} \pi^{2}-4 \bar{R} \bar{\omega}^{2}} / 2, \quad \eta_{m}=\phi \sqrt{m^{2} \pi^{2}-4 \bar{S} \bar{\omega}^{2}} / 2, \quad \epsilon_{m}=m^{2} \pi^{2} \bar{h}^{2} \quad-20 \delta \bar{R} \bar{\omega}^{2}$, $\zeta_{m}=m^{2} \pi^{2} \bar{h}^{2}-20 \delta \bar{S} \bar{\omega}^{2}$, and $\gamma_{m}=\phi \sqrt{40 / \bar{h}^{2}+m^{2} \pi^{2}} / 2$, with $\bar{h}=h / a$. The coordinates exchange gives the solutions of the second fundamental problem (Fig. $1(c)$ ) by replacing $x, y, a, b, E_{m}$, and $F_{m}$ with $y, x, b, a, G_{m}$, and $H_{m}$, respectively.

\section{Natural Frequency and Mode Shape Solutions by Superposition}

For convenience, we use "S", "C", "F," and "SC" to stand for the simply supported, clamped, free and slidingly clamped edges, respectively. A clockwise four-letter symbolic notation is applied to a plate, with $y=b$ as the starting edge. The most complex free vibration problem of the rectangular thick plates with a free corner is for CCFF plate. It will be seen later that the other plates with a free corner, including CSFF and SSFF plates, can be treated as the special cases of CCFF plate when applying further simplifications. Superposition of the two fundamental solutions given in Sec. 2 constitutes the solution of a CCFF plate, as revealed in Fig. 1. However, there exist four sets of constants needing to be determined. To reach equivalence between Figs. 1 $(a)$ and $1(b)$ plus $1(c)$, the conditions that the following quantities vanish must be enforced: (a) superposition of $M_{y}$ of the two fundamental problems at $y=0$; (b) superposition of $\psi_{y}$ at $y=b ;(\mathbf{c})$ superposition of $M_{x}$ at $x=0$; and (d) superposition of $\psi_{x}$ at $x=a$. Corresponding to condition (a), we have

$$
\begin{aligned}
& {\left[\phi^{2}\left(\xi_{i} \zeta_{i} \bar{\epsilon}_{i} \text { th } \eta_{i}-\eta_{i} \epsilon_{i} \bar{\zeta}_{i} \text { th } \xi_{i}\right)+4 \delta \xi_{i} \eta_{i} \gamma_{i} i^{2} \pi^{2} \bar{h}^{2} \bar{\omega}^{2} \text { th } \gamma_{i}(1-\nu)(\bar{R}-\bar{S})\right] E_{i}} \\
& \quad+20 \delta \phi \xi_{i} \eta_{i}\left[\bar{\epsilon}_{i} \operatorname{sech} \eta_{i}-\bar{\zeta}_{i} \operatorname{sech} \xi_{i}+\delta i^{2} \pi^{2} \bar{\omega}^{2} \operatorname{sech} \gamma_{i}(1-\nu)(\bar{R}-\bar{S})\right] \bar{F}_{i} \\
& \quad-\sum_{m=1,3,5, \ldots}^{\infty} \frac{32 \phi \xi_{i} \eta_{i} \bar{\omega}^{2}(\bar{R}-\bar{S})}{\left(4 \xi_{i}^{2}+m^{2} \pi^{2}\right)\left(4 \eta_{i}^{2}+m^{2} \pi^{2}\right)\left[40 \phi^{2}+\pi^{2} \bar{h}^{2}\left(m^{2}+i^{2} \phi^{2}\right)\right]} \\
& \quad \times\left\{\left\{i^{2} \pi^{2} \phi^{2}\left\{4 \delta \bar{R} \bar{S} \phi^{2} \bar{h}^{2} \bar{\omega}^{4}\left[20 \nu \phi^{2}-m^{2} \pi^{2}(1-\nu)\left(\bar{h}^{2}-5 \delta\right)\right]+m^{2} \pi^{2}\left\{m^{2} \pi^{2} \bar{h}^{4}(1+\nu)-4 \phi^{2}\left[100 \delta(1-\nu)+\bar{\omega}^{2} \bar{h}^{4}(\bar{R}+\bar{S})\right]\right\}\right\}\right.\right. \\
& \quad+4 \bar{h}^{2}\left\{i^{2} \pi^{2} \phi^{2} \gamma_{i}^{2}\left\{m^{2} \pi^{2}\left[\nu \bar{h}^{2}+5 \delta(1-\nu)\right]+20 \delta \phi^{2} \bar{\omega}^{2}(1-\nu)\left(\bar{R}+\bar{S}+\delta \bar{R} \bar{S} \bar{\omega}^{2}\right)\right\}\right. \\
& \left.\left.+\bar{\gamma}_{i}^{2}\left\{80 \nu \delta \bar{R} \bar{S} \phi^{4} \bar{\omega}^{4}+m^{2} \pi^{2}\left[\bar{h}^{2}-5 \delta(1-\nu)\right]\left[m^{2} \pi^{2}-4 \phi^{2} \bar{\omega}^{2}(\bar{R}+\bar{S})\right]\right\}\right\}\right\} G_{m} \\
& +10 i \pi \delta \phi^{2} \sin \left(\frac{i \pi}{2}\right)\left\{4 \gamma_{i}^{2} \bar{h}^{2}\left\{4\left(\xi_{i}^{2}+\eta_{i}^{2}\right)-\phi^{2}\left[i^{2} \pi^{2}(2-\nu)+4 \delta \bar{R} \bar{S} \bar{\omega}^{4}(1-\nu)\right]\right\}+m^{2} \pi^{2}\left\{\phi^{2}\left(40+\nu i^{2} \pi^{2} \bar{h}^{2}\right)+\left[\bar{h}^{2}-10 \delta(1-\nu)\right]\right.\right. \\
& \left.\left.\left.\quad \times\left[4\left(\xi_{i}^{2}+\eta_{i}^{2}\right)+\pi^{2}\left(m^{2}-i^{2} \phi^{2}\right)\right]\right\}\right\} \bar{H}_{m}\right\}=0
\end{aligned}
$$


for $i=1,3,5, \ldots$, where $\bar{F}_{i}=a F_{i} / D, \quad \bar{H}_{m}=a H_{m} / D, \quad \xi_{i}=\phi \sqrt{i^{2} \pi^{2}-4 \bar{R} \bar{\omega}^{2}} / 2, \quad \eta_{i}=\phi \sqrt{i^{2} \pi^{2}-4 \bar{S} \bar{\omega}^{2}} / 2, \quad \epsilon_{i}=i^{2} \pi^{2} \bar{h}^{2}-20 \delta \bar{R} \bar{\omega}^{2}$,

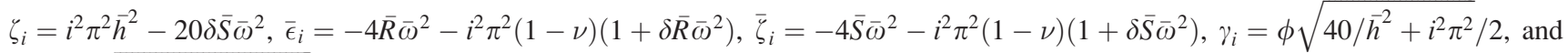
$\bar{\gamma}_{i}=\phi \sqrt{40 / \bar{h}^{2}+i^{2} \pi^{2} / \phi^{2}} / 2$. Corresponding to condition (b), we have

$$
\begin{aligned}
& \phi \gamma_{i}\left[\delta i^{2} \pi^{2} \bar{h}^{2} \bar{\omega}^{2} \operatorname{sech} \gamma_{i}(\bar{R}-\bar{S})+\epsilon_{i} \operatorname{sech} \xi_{i}\left(1+\delta \bar{S} \bar{\omega}^{2}\right)-\zeta_{i} \operatorname{sech} \eta_{i}\left(1+\delta \bar{R} \bar{\omega}^{2}\right)\right] E_{i} \\
& \quad-5 \delta\left\{\delta i^{2} \pi^{2} \phi^{2} \bar{\omega}^{2} \text { th } \gamma_{i}(\bar{R}-\bar{S})+4 \gamma_{i}\left[\xi_{i} \text { th } \xi_{i}\left(1+\delta \bar{S} \bar{\omega}^{2}\right)-\eta_{i} \text { th } \eta_{i}\left(1+\delta \bar{R} \bar{\omega}^{2}\right)\right]\right\} \bar{F}_{i} \\
& \quad+\sum_{m=1,3,5, \ldots}^{\infty} \sin \left(\frac{m \pi}{2}\right) \frac{16 m \pi \gamma_{i} \phi^{2} \bar{\omega}^{2}(\bar{R}-\bar{S})}{\left(4 \xi_{i}^{2}+m^{2} \pi^{2}\right)\left(4 \eta_{i}^{2}+m^{2} \pi^{2}\right)\left[40 \phi^{2}+\pi^{2} \bar{h}^{2}\left(m^{2}+i^{2} \phi^{2}\right)\right]} \\
& \quad \times\left\{\left\{\pi^{2}\left\{\pi^{2} \bar{h}^{2} m^{4}\left(\bar{h}^{2}-5 \delta\right)-5 \delta i^{2} \phi^{4}\left(40-i^{2} \pi^{2} \bar{h}^{2}\right)-m^{2} \phi^{2}\left[200 \delta-\bar{h}^{2}\left(40+i^{2} \pi^{2} \bar{h}^{2}\right)\right]\right\}\right.\right. \\
& \left.\quad-40 \delta i^{2} \pi^{2} \bar{h}^{2} \bar{\omega}^{2} \phi^{4}(\bar{R}+\bar{S})-4 \delta \bar{R} \bar{S} \phi^{2}\left\{m^{2} \pi^{2} \bar{h}^{2}\left(5 \delta-\bar{h}^{2}\right)+5 \phi^{2} \bar{\omega}^{4}\left[40 \delta-\bar{h}^{2}\left(8-\delta i^{2} \pi^{2}\right)\right]\right\}\right\} G_{m} \\
& \left.\quad+10 \delta i \pi \phi^{2} \sin \left(\frac{i \pi}{2}\right)\left\{\pi^{2}\left(\bar{h}^{2}-10 \delta\right)\left(m^{2}+i^{2} \phi^{2}\right)+4 \phi^{2}\left\{10+\delta \bar{\omega}^{2}\left[\bar{R} \bar{S} \bar{h}^{2} \bar{\omega}^{2}+10(\bar{R}+\bar{S})\right]\right\}\right\} \bar{H}_{m}\right\}=0
\end{aligned}
$$

for $i=1,3,5, \ldots$ Corresponding to conditions (c) and (d), one just needs to replace $a, b, E_{i}, F_{i}, G_{m}$, and $H_{m}$ in Eqs. (22) and (23) with $b, a, G_{i}, H_{i}, E_{m}$, and $F_{m}$, respectively, to yield two more sets of equations. The generated four sets of linear equations with respect to four sets of unknowns $E_{m}, F_{m}, G_{n}$, and $H_{n}$ ( $m=1,3,5, \ldots ; n=1,3,5, \ldots)$ have nonzero solutions provided that the determinant of the equations' coefficient matrix is zero. Because the frequency parameter $\bar{\omega}$ has been included in the coefficient matrix, the zero-determinant condition will yield an equation with respect to $\bar{\omega}$. The mode shape corresponding to a frequency can be obtained by seeking a set of nonzero solutions for $E_{m}, F_{m}, G_{n}$, and $H_{n}$ under one determined $\bar{\omega}$, followed by their substitution into two fundamental problems' solutions and summation. It is worth pointing out that the solution for CCFF plate

\begin{tabular}{|c|c|c|c|c|c|c|c|c|c|c|c|}
\hline \multirow[b]{2}{*}{$b / a$} & \multirow[b]{2}{*}{ Reference } & \multicolumn{10}{|c|}{ Mode } \\
\hline & & First & Second & Third & Fourth & Fifth & Sixth & Seventh & Eighth & Ninth & Tenth \\
\hline \multirow[t]{3}{*}{1} & Present & 6.8327 & 23.418 & 26.096 & 45.966 & 60.760 & 63.513 & 81.574 & 84.255 & 115.24 & 117.68 \\
\hline & FEM (shell) & 6.8273 & 23.329 & 26.011 & 45.711 & 60.283 & 63.045 & 80.777 & 83.497 & 113.73 & 116.19 \\
\hline & FEM (brick) & 6.7010 & 22.902 & 25.541 & 44.908 & 59.235 & 61.957 & 79.401 & 82.102 & 111.90 & 114.34 \\
\hline \multirow[t]{3}{*}{1.5} & Present & 4.9237 & 13.037 & 22.924 & 29.544 & 33.265 & 50.464 & 54.552 & 60.871 & 70.098 & 75.256 \\
\hline & FEM (shell) & 4.9208 & 13.013 & 22.846 & 29.430 & 33.131 & 50.166 & 54.200 & 60.420 & 69.523 & 74.632 \\
\hline & FEM (brick) & 4.8284 & 12.771 & 22.427 & 28.889 & 32.535 & 49.277 & 53.236 & 59.372 & 68.323 & 73.344 \\
\hline \multirow[t]{3}{*}{2} & Present & 4.2515 & 8.9751 & 18.083 & 22.415 & 28.182 & 32.307 & 38.637 & 50.910 & 53.055 & 60.380 \\
\hline & FEM (shell) & 4.2493 & 8.9648 & 18.040 & 22.342 & 28.076 & 32.181 & 38.460 & 50.609 & 52.739 & 59.935 \\
\hline & FEM (brick) & 4.1691 & 8.7965 & 17.702 & 21.931 & 27.566 & 31.587 & 37.768 & 49.696 & 51.800 & 58.895 \\
\hline \multirow[t]{3}{*}{2.5} & Present & 3.9523 & 7.0476 & 12.870 & 21.417 & 22.494 & 25.911 & 32.681 & 34.036 & 42.358 & 48.912 \\
\hline & FEM (shell) & 3.9503 & 7.0416 & 12.848 & 21.353 & 22.427 & 25.819 & 32.546 & 33.902 & 42.149 & 48.641 \\
\hline & FEM (brick) & 3.8756 & 6.9090 & 12.607 & 20.954 & 22.012 & 25.347 & 31.954 & 33.276 & 41.390 & 47.758 \\
\hline \multirow[t]{3}{*}{3} & Present & 3.7988 & 5.9726 & 10.022 & 16.151 & 21.945 & 24.179 & 25.018 & 29.511 & 34.995 & 36.435 \\
\hline & FEM (shell) & 3.7969 & 5.9682 & 10.009 & 16.119 & 21.873 & 24.099 & 24.940 & 29.398 & 34.853 & 36.277 \\
\hline & FEM (brick) & 3.7250 & 5.8557 & 9.8200 & 15.814 & 21.471 & 23.652 & 24.479 & 28.862 & 34.207 & 35.619 \\
\hline \multirow[t]{3}{*}{3.5} & Present & 3.7113 & 5.3112 & 8.2993 & 12.784 & 18.826 & 21.928 & 23.833 & 26.506 & 27.628 & 32.634 \\
\hline & FEM (shell) & 3.7095 & 5.3077 & 8.2907 & 12.764 & 18.782 & 21.856 & 23.752 & 26.420 & 27.530 & 32.501 \\
\hline & FEM (brick) & 3.6392 & 5.2076 & 8.1342 & 12.522 & 18.427 & 21.454 & 23.316 & 25.925 & 27.026 & 31.910 \\
\hline \multirow[t]{3}{*}{4} & Present & 3.6574 & 4.8772 & 7.1761 & 10.598 & 15.219 & 20.942 & 21.979 & 23.365 & 26.152 & 28.201 \\
\hline & FEM (shell) & 3.6556 & 4.8742 & 7.1697 & 10.584 & 15.190 & 20.887 & 21.910 & 23.287 & 26.058 & 28.109 \\
\hline & FEM (brick) & 3.5863 & 4.7821 & 7.0340 & 10.383 & 14.902 & 20.493 & 21.506 & 22.859 & 25.581 & 27.581 \\
\hline \multirow[t]{3}{*}{4.5} & Present & 3.6221 & 4.5787 & 6.4017 & 9.1002 & 12.736 & 17.326 & 21.775 & 22.728 & 23.260 & 25.260 \\
\hline & FEM (shell) & 3.6202 & 4.5760 & 6.3963 & 9.0899 & 12.716 & 17.289 & 21.704 & 22.658 & 23.191 & 25.172 \\
\hline & FEM (brick) & 3.5516 & 4.4896 & 6.2756 & 8.9177 & 12.474 & 16.961 & 21.304 & 22.236 & 22.760 & 24.711 \\
\hline \multirow[t]{3}{*}{5} & Present & 3.5977 & 4.3659 & 5.8449 & 8.0294 & 10.962 & 14.670 & 19.138 & 21.817 & 22.751 & 24.296 \\
\hline & FEM (shell) & 3.5959 & 4.3634 & 5.8407 & 8.0217 & 10.947 & 14.644 & 19.095 & 21.745 & 22.675 & 24.219 \\
\hline & FEM (brick) & 3.5277 & 4.2809 & 5.7302 & 7.8691 & 10.739 & 14.365 & 18.732 & 21.345 & 22.259 & 23.767 \\
\hline
\end{tabular}
can reduce to those for CSFF and SSFF plates. Simply deleting the set of equations corresponding to condition (d) and setting $H_{n}=0$, the solution of a CSFF plate can be obtained. Deleting the equations corresponding to both conditions (b) and (d) and

Table 1 Frequency parameters, $\omega a^{2} \sqrt{\rho h / D}$, of CCFF thick plate with $h / a=0.05$ 
Table 2 Frequency parameters, $\omega a^{2} \sqrt{\rho h / D}$, of CCFF thick plate with $h / a=0.1$

\begin{tabular}{|c|c|c|c|c|c|c|c|c|c|c|c|}
\hline \multirow[b]{2}{*}{$b / a$} & \multirow[b]{2}{*}{ Reference } & \multicolumn{10}{|c|}{ Mode } \\
\hline & & First & Second & Third & Fourth & Fifth & Sixth & Seventh & Eighth & Ninth & Tenth \\
\hline \multirow[t]{4}{*}{1} & Present & 6.6934 & 22.457 & 25.010 & 42.797 & 56.302 & 58.768 & 73.632 & 76.064 & 101.95 & 103.84 \\
\hline & FEM (shell) & 6.6727 & 22.143 & 24.722 & 41.999 & 54.905 & 57.425 & 71.452 & 74.072 & 98.344 & 100.33 \\
\hline & FEM (brick) & 6.6564 & 22.106 & 24.695 & 41.976 & 54.929 & 57.467 & 71.503 & 74.173 & 98.608 & 100.59 \\
\hline & Liew et al. [30] & 6.6738 & 22.145 & 24.722 & 42.002 & 54.908 & 57.429 & 71.455 & 74.076 & 98.352 & 100.34 \\
\hline \multirow[t]{4}{*}{1.5} & Present & 4.8528 & 12.676 & 22.099 & 28.300 & 31.540 & 46.992 & 51.080 & 56.500 & 64.302 & 68.664 \\
\hline & FEM (shell) & 4.8416 & 12.589 & 21.828 & 27.914 & 31.097 & 46.076 & 50.019 & 55.191 & 62.679 & 66.935 \\
\hline & FEM (brick) & 4.8282 & 12.558 & 21.797 & 27.866 & 31.063 & 46.047 & 50.005 & 55.228 & 62.726 & 66.960 \\
\hline & Liew et al. [30] & 4.8420 & 12.591 & 21.831 & 27.916 & 31.099 & 46.080 & 50.022 & 55.196 & 62.684 & 66.943 \\
\hline \multirow[t]{4}{*}{2} & Present & 4.2047 & 8.7739 & 17.547 & 21.643 & 26.920 & 30.947 & 36.449 & 47.916 & 49.428 & 56.097 \\
\hline & FEM (shell) & 4.1958 & 8.7349 & 17.392 & 21.385 & 26.562 & 30.526 & 35.876 & 46.988 & 48.468 & 54.801 \\
\hline & FEM (brick) & 4.1840 & 8.7104 & 17.348 & 21.355 & 26.525 & 30.472 & 35.837 & 46.950 & 48.440 & 54.839 \\
\hline & Liew et al. [30] & 4.1966 & 8.7366 & 17.395 & 21.386 & 26.565 & 30.530 & 35.880 & 46.998 & 48.479 & 54.809 \\
\hline \multirow[t]{4}{*}{2.5} & Present & 3.9162 & 6.9125 & 12.554 & 20.731 & 21.746 & 24.851 & 31.071 & 32.577 & 39.858 & 46.205 \\
\hline & FEM (shell) & 3.9083 & 6.8889 & 12.477 & 20.502 & 21.512 & 24.535 & 30.624 & 32.132 & 39.194 & 45.366 \\
\hline & FEM (brick) & 3.8973 & 6.8688 & 12.440 & 20.457 & 21.475 & 24.501 & 30.582 & 32.075 & 39.152 & 45.319 \\
\hline & Liew et al. [30] & 3.9084 & 6.8929 & 12.479 & 20.506 & 21.513 & 24.539 & 30.629 & 32.139 & 39.206 & 45.384 \\
\hline \multirow[t]{3}{*}{3} & Present & 3.7683 & 5.8725 & 9.8047 & 15.727 & 21.218 & 23.312 & 24.119 & 28.162 & 33.497 & 34.519 \\
\hline & FEM (shell) & 3.7609 & 5.8557 & 9.7578 & 15.609 & 20.965 & 23.032 & 23.854 & 27.782 & 33.026 & 34.005 \\
\hline & FEM (brick) & 3.7504 & 5.8383 & 9.7283 & 15.564 & 20.935 & 22.988 & 23.809 & 27.743 & 32.964 & 33.961 \\
\hline \multirow[t]{3}{*}{3.5} & Present & 3.6843 & 5.2321 & 8.1359 & 12.488 & 18.301 & 21.204 & 22.955 & 25.561 & 26.478 & 31.038 \\
\hline & FEM (shell) & 3.6771 & 5.2188 & 8.1041 & 12.413 & 18.145 & 20.954 & 22.674 & 25.262 & 26.149 & 30.596 \\
\hline & FEM (brick) & 3.6669 & 5.2032 & 8.0789 & 12.375 & 18.092 & 20.924 & 22.641 & 25.204 & 26.109 & 30.553 \\
\hline \multirow[t]{3}{*}{4} & Present & 3.6325 & 4.8119 & 7.0458 & 10.373 & 14.844 & 20.322 & 21.263 & 22.526 & 25.091 & 27.192 \\
\hline & FEM (shell) & 3.6255 & 4.8004 & 7.0221 & 10.322 & 14.742 & 20.125 & 21.022 & 22.254 & 24.771 & 26.876 \\
\hline & FEM (brick) & 3.6154 & 4.7862 & 7.0001 & 10.289 & 14.696 & 20.071 & 20.990 & 22.223 & 24.736 & 26.812 \\
\hline \multirow[t]{3}{*}{4.5} & Present & 3.5986 & 4.5230 & 6.2937 & 8.9204 & 12.450 & 16.877 & 21.066 & 21.979 & 22.491 & 24.271 \\
\hline & FEM (shell) & 3.5918 & 4.5127 & 6.2745 & 8.8825 & 12.377 & 16.747 & 20.818 & 21.730 & 22.256 & 23.968 \\
\hline & FEM (brick) & 3.5818 & 4.4994 & 6.2550 & 8.8536 & 12.338 & 16.696 & 20.787 & 21.688 & 22.209 & 23.933 \\
\hline \multirow[t]{3}{*}{5} & Present & 3.5754 & 4.3170 & 5.7528 & 7.8799 & 10.733 & 14.324 & 18.619 & 21.104 & 21.965 & 23.451 \\
\hline & FEM (shell) & 3.5685 & 4.3076 & 5.7367 & 7.8502 & 10.679 & 14.230 & 18.463 & 20.855 & 21.701 & 23.176 \\
\hline & FEM (brick) & 3.5587 & 4.2949 & 5.7190 & 7.8251 & 10.644 & 14.184 & 18.407 & 20.826 & 21.671 & 23.130 \\
\hline
\end{tabular}

Table 3 Frequency parameters, $\omega a^{2} \sqrt{\rho h / D}$, of CCFF thick plate with $h / a=0.2$

\begin{tabular}{|c|c|c|c|c|c|c|c|c|c|c|c|}
\hline \multirow[b]{2}{*}{$b / a$} & \multirow[b]{2}{*}{ Reference } & \multicolumn{10}{|c|}{ Mode } \\
\hline & & First & Second & Third & Fourth & Fifth & Sixth & Seventh & Eighth & Ninth & Tenth \\
\hline \multirow[t]{4}{*}{1} & Present & 6.3126 & 19.806 & 21.921 & 35.427 & 45.438 & 47.208 & 57.159 & 58.733 & 75.425 & 76.528 \\
\hline & FEM (shell) & 6.2445 & 18.969 & 21.218 & 33.770 & 42.900 & 44.939 & 53.567 & 55.802 & 70.541 & 72.030 \\
\hline & FEM (brick) & 6.2661 & 19.073 & 21.367 & 34.014 & 43.313 & 45.394 & 54.074 & 56.407 & 71.434 & 72.929 \\
\hline & Liew et al. [30] & 6.2455 & 18.970 & 21.219 & 33.771 & 42.901 & 44.939 & 53.568 & 55.802 & 70.545 & 72.034 \\
\hline \multirow[t]{4}{*}{1.5} & Present & 4.6522 & 11.656 & 19.622 & 24.806 & 27.085 & 38.794 & 42.131 & 45.624 & 50.999 & 54.149 \\
\hline & FEM (shell) & 4.6132 & 11.392 & 18.919 & 23.855 & 26.076 & 36.982 & 40.146 & 43.332 & 48.292 & 51.343 \\
\hline & FEM (brick) & 4.6269 & 11.434 & 19.040 & 23.992 & 26.254 & 37.256 & 40.492 & 43.776 & 48.783 & 51.827 \\
\hline & Liew et al. [30] & 4.6140 & 11.392 & 18.920 & 23.857 & 26.077 & 36.983 & 40.148 & 43.333 & 48.293 & 51.345 \\
\hline \multirow[t]{4}{*}{2} & Present & 4.0663 & 8.2084 & 15.989 & 19.259 & 23.483 & 27.073 & 30.945 & 40.013 & 40.747 & 45.352 \\
\hline & FEM (shell) & 4.0351 & 8.0814 & 15.551 & 18.585 & 22.604 & 26.090 & 29.693 & 38.206 & 38.939 & 43.079 \\
\hline & FEM (brick) & 4.0471 & 8.1059 & 15.612 & 18.707 & 22.748 & 26.242 & 29.895 & 38.495 & 39.249 & 43.524 \\
\hline & Liew et al. [30] & 4.0357 & 8.0822 & 15.553 & 18.586 & 22.605 & 26.091 & 29.694 & 38.208 & 38.941 & 43.081 \\
\hline \multirow[t]{4}{*}{2.5} & Present & 3.8050 & 6.5320 & 11.644 & 18.658 & 19.465 & 21.842 & 26.817 & 28.439 & 33.622 & 38.899 \\
\hline & FEM (shell) & 3.7769 & 6.4535 & 11.405 & 18.019 & 18.875 & 21.051 & 25.773 & 27.428 & 32.223 & 37.273 \\
\hline & FEM (brick) & 3.7883 & 6.4711 & 11.440 & 18.114 & 18.981 & 21.186 & 25.938 & 27.590 & 32.446 & 37.550 \\
\hline & Liew et al. [30] & 3.7771 & 6.4537 & 11.406 & 18.020 & 18.877 & 21.052 & 25.773 & 27.430 & 32.225 & 37.277 \\
\hline \multirow[t]{3}{*}{3} & Present & 3.6711 & 5.5888 & 9.1833 & 14.478 & 18.930 & 20.719 & 21.523 & 24.508 & 29.179 & 29.604 \\
\hline & FEM (shell) & 3.6445 & 5.5315 & 9.0327 & 14.134 & 18.265 & 19.973 & 20.877 & 23.588 & 28.067 & 28.507 \\
\hline & FEM (brick) & 3.6557 & 5.5462 & 9.0572 & 14.181 & 18.386 & 20.092 & 20.984 & 23.739 & 28.237 & 28.688 \\
\hline \multirow[t]{3}{*}{3.5} & Present & 3.5951 & 5.0061 & 7.6700 & 11.621 & 16.740 & 18.915 & 18.915 & 22.733 & 23.311 & 26.814 \\
\hline & FEM (shell) & 3.5694 & 4.9598 & 7.5643 & 11.393 & 16.300 & 18.258 & 19.612 & 21.927 & 22.562 & 25.781 \\
\hline & FEM (brick) & 3.5806 & 4.9730 & 7.5832 & 11.425 & 16.358 & 18.381 & 19.741 & 22.046 & 22.689 & 25.947 \\
\hline \multirow[t]{3}{*}{4} & Present & 3.5486 & 4.6230 & 6.6748 & 9.7216 & 13.735 & 18.441 & 19.018 & 19.989 & 22.067 & 24.213 \\
\hline & FEM (shell) & 3.5234 & 4.5831 & 6.5942 & 9.5586 & 13.432 & 17.870 & 18.416 & 19.288 & 21.266 & 23.421 \\
\hline & FEM (brick) & 3.5345 & 4.5955 & 6.6105 & 9.5825 & 13.472 & 17.953 & 18.525 & 19.415 & 21.403 & 23.535 \\
\hline \multirow[t]{3}{*}{4.5} & Present & 3.5182 & 4.3595 & 5.9859 & 8.4021 & 11.609 & 15.534 & 18.817 & 19.631 & 20.237 & 21.405 \\
\hline & FEM (shell) & 3.4934 & 4.3235 & 5.9210 & 8.2787 & 11.386 & 15.162 & 18.161 & 18.944 & 19.657 & 20.640 \\
\hline & FEM (brick) & 3.5045 & 4.3355 & 5.9354 & 8.2982 & 11.416 & 15.210 & 18.281 & 19.063 & 19.746 & 20.773 \\
\hline \multirow[t]{3}{*}{5} & Present & 3.4974 & 4.1716 & 5.4898 & 7.4506 & 10.063 & 13.293 & 17.058 & 18.837 & 19.537 & 20.832 \\
\hline & FEM (shell) & 3.4728 & 4.1384 & 5.4347 & 7.3526 & 9.8916 & 13.012 & 16.621 & 18.184 & 18.851 & 20.088 \\
\hline & FEM (brick) & 3.4840 & 4.1501 & 5.4482 & 7.3695 & 9.9155 & 13.049 & 16.677 & 18.306 & 18.978 & 20.214 \\
\hline
\end{tabular}


Table 4 Frequency parameters, $\omega a^{2} \sqrt{\rho h / D}$, of CSFF thick plate with $h / a=0.2$

\begin{tabular}{|c|c|c|c|c|c|c|c|c|c|c|c|}
\hline \multirow[b]{2}{*}{$b / a$} & \multirow[b]{2}{*}{ Reference } & \multicolumn{10}{|c|}{ Mode } \\
\hline & & First & Second & Third & Fourth & Fifth & Sixth & Seventh & Eighth & Ninth & Tenth \\
\hline \multirow[t]{4}{*}{1} & Present & 4.9666 & 16.927 & 20.685 & 33.556 & 42.155 & 46.323 & 55.319 & 57.284 & 73.039 & 75.829 \\
\hline & FEM (shell) & 4.9173 & 16.272 & 19.955 & 31.981 & 39.667 & 43.944 & 51.915 & 54.187 & 68.160 & 71.088 \\
\hline & FEM (brick) & 4.9290 & 16.302 & 20.041 & 32.151 & 39.880 & 44.368 & 52.287 & 54.694 & 68.771 & 71.829 \\
\hline & Liew et al. [30] & 4.9180 & 16.272 & 19.956 & 31.981 & 39.667 & 43.944 & 51.916 & 54.188 & 68.162 & 71.088 \\
\hline \multirow[t]{4}{*}{1.5} & Present & 2.9049 & 10.885 & 15.627 & 23.925 & 24.933 & 37.187 & 41.090 & 42.351 & 47.771 & 53.234 \\
\hline & FEM (shell) & 2.8846 & 10.638 & 15.077 & 22.947 & 24.065 & 35.443 & 38.741 & 40.368 & 45.046 & 50.461 \\
\hline & FEM (brick) & 2.8877 & 10.667 & 15.100 & 23.029 & 24.199 & 35.645 & 38.959 & 40.700 & 45.335 & 50.883 \\
\hline & Liew et al. [30] & 2.8849 & 10.638 & 15.077 & 22.948 & 24.066 & 35.444 & 38.741 & 40.369 & 45.047 & 50.462 \\
\hline \multirow[t]{4}{*}{2} & Present & 2.0272 & 7.1681 & 14.762 & 15.825 & 20.290 & 26.774 & 28.699 & 39.103 & 39.831 & 41.310 \\
\hline & FEM (shell) & 2.0151 & 7.0610 & 14.253 & 15.389 & 19.536 & 25.793 & 27.532 & 37.272 & 37.930 & 39.151 \\
\hline & FEM (brick) & 2.0160 & 7.0719 & 14.275 & 15.437 & 19.587 & 25.933 & 27.653 & 37.489 & 38.190 & 39.403 \\
\hline & Liew et al. [30] & 2.0154 & 7.0617 & 14.254 & 15.391 & 19.536 & 25.794 & 27.533 & 37.273 & 37.932 & 39.151 \\
\hline \multirow[t]{4}{*}{2.5} & Present & 1.5456 & 5.2678 & 10.906 & 14.806 & 18.126 & 18.907 & 24.140 & 28.149 & 31.626 & 38.693 \\
\hline & FEM (shell) & 1.5369 & 5.2086 & 10.685 & 14.285 & 17.478 & 18.356 & 23.203 & 27.140 & 30.297 & 37.031 \\
\hline & FEM (brick) & 1.5371 & 5.2130 & 10.708 & 14.302 & 17.518 & 18.423 & 23.279 & 27.291 & 30.438 & 37.293 \\
\hline & Liew et al. [30] & 1.5367 & 5.2092 & 10.686 & 14.285 & 17.478 & 18.357 & 23.203 & 27.141 & 30.298 & 37.036 \\
\hline \multirow[t]{3}{*}{3} & Present & 1.2441 & 4.1403 & 8.2748 & 13.800 & 17.160 & 20.898 & 21.565 & 27.066 & 29.074 & 33.709 \\
\hline & FEM (shell) & 1.2373 & 4.1022 & 8.1436 & 13.442 & 16.544 & 20.231 & 20.803 & 25.983 & 28.042 & 32.272 \\
\hline & FEM (brick) & 1.2372 & 4.1039 & 8.1547 & 13.471 & 16.573 & 20.304 & 20.868 & 26.082 & 28.198 & 32.432 \\
\hline \multirow[t]{3}{*}{3.5} & Present & 1.0390 & 3.4002 & 6.6014 & 10.899 & 14.584 & 16.153 & 16.667 & 19.743 & 22.659 & 24.143 \\
\hline & FEM (shell) & 1.0335 & 3.3731 & 6.5150 & 10.688 & 14.074 & 15.645 & 16.175 & 19.014 & 21.961 & 23.219 \\
\hline & FEM (brick) & 1.0332 & 3.3734 & 6.5208 & 10.707 & 14.090 & 15.676 & 16.218 & 19.058 & 22.054 & 23.294 \\
\hline \multirow[t]{3}{*}{4} & Present & 0.89116 & 2.8794 & 5.4641 & 8.8735 & 13.101 & 14.604 & 15.988 & 18.157 & 18.685 & 22.074 \\
\hline & FEM (shell) & 0.88649 & 2.8585 & 5.4032 & 8.7292 & 12.806 & 14.102 & 15.423 & 17.611 & 18.070 & 21.240 \\
\hline & FEM (brick) & 0.88617 & 2.8583 & 5.4058 & 8.7404 & 12.833 & 14.119 & 15.446 & 17.658 & 18.119 & 21.299 \\
\hline \multirow[t]{3}{*}{4.5} & Present & 0.77980 & 2.4941 & 4.6487 & 7.4324 & 10.895 & 14.450 & 15.078 & 15.729 & 17.757 & 19.766 \\
\hline & FEM (shell) & 0.77578 & 2.4773 & 4.6030 & 7.3281 & 10.690 & 13.957 & 14.684 & 15.199 & 17.118 & 19.203 \\
\hline & FEM (brick) & 0.77541 & 2.4768 & 4.6041 & 7.3344 & 10.708 & 13.974 & 14.718 & 15.225 & 17.151 & 19.268 \\
\hline \multirow[t]{3}{*}{5} & Present & 0.69303 & 2.1983 & 4.0389 & 6.3681 & 9.2496 & 12.660 & 14.528 & 15.425 & 16.635 & 17.209 \\
\hline & FEM (shell) & 0.68945 & 2.1843 & 4.0031 & 6.2895 & 9.0974 & 12.392 & 14.024 & 14.882 & 16.193 & 16.623 \\
\hline & FEM (brick) & 0.68910 & 2.1835 & 4.0032 & 6.2928 & 9.1084 & 12.416 & 14.041 & 14.903 & 16.234 & 16.657 \\
\hline
\end{tabular}

Table 5 Frequency parameters, $\omega a^{2} \sqrt{\rho h / D}$, of SSFF thick plate with $h / a=0.2$

\begin{tabular}{|c|c|c|c|c|c|c|c|c|c|c|c|}
\hline \multirow[b]{2}{*}{$b / a$} & \multirow[b]{2}{*}{ Reference } & \multicolumn{10}{|c|}{ Mode } \\
\hline & & First & Second & Third & Fourth & Fifth & Sixth & Seventh & Eighth & Ninth & Tenth \\
\hline \multirow[t]{4}{*}{1} & Present & 3.2081 & 15.787 & 17.510 & 31.590 & 41.359 & 42.960 & 54.182 & 55.152 & 72.478 & 73.642 \\
\hline & FEM (shell) & 3.1797 & 15.158 & 16.918 & 30.099 & 38.797 & 40.610 & 50.777 & 52.093 & 67.463 & 68.977 \\
\hline & FEM (brick) & 3.1767 & 15.167 & 16.945 & 30.186 & 38.981 & 40.840 & 51.072 & 52.436 & 68.044 & 69.584 \\
\hline & Liew et al. [30] & 3.1800 & 15.158 & 16.917 & 30.099 & 38.797 & 40.610 & 50.777 & 52.093 & 67.462 & 68.977 \\
\hline \multirow[t]{4}{*}{1.5} & Present & 2.1459 & 8.9337 & 15.414 & 21.730 & 23.418 & 35.723 & 39.164 & 41.517 & 47.414 & 51.605 \\
\hline & FEM (shell) & 2.1318 & 8.7475 & 14.861 & 20.881 & 22.516 & 34.025 & 37.122 & 39.222 & 44.675 & 48.861 \\
\hline & FEM (brick) & 2.1297 & 8.7475 & 14.877 & 20.928 & 22.571 & 34.159 & 37.314 & 39.439 & 44.938 & 49.175 \\
\hline & Liew et al. [30] & 2.1318 & 8.7474 & 14.861 & 20.881 & 22.517 & 34.025 & 37.122 & 39.222 & 44.675 & 48.861 \\
\hline \multirow[t]{4}{*}{2} & Present & 1.6018 & 6.0129 & 13.524 & 15.127 & 19.795 & 24.659 & 27.801 & 37.755 & 38.079 & 41.169 \\
\hline & FEM (shell) & 1.5924 & 5.9297 & 13.140 & 14.617 & 19.051 & 23.727 & 26.651 & 35.969 & 36.295 & 38.867 \\
\hline & FEM (brick) & 1.5908 & 5.9275 & 13.152 & 14.636 & 19.085 & 23.802 & 26.733 & 36.146 & 36.473 & 39.082 \\
\hline & Liew et al. [30] & 1.5920 & 5.9297 & 13.140 & 14.618 & 19.05 & 23.728 & 26.652 & 35.969 & 36.295 & 38.867 \\
\hline \multirow[t]{4}{*}{2.5} & Present & 1.2746 & 4.5144 & 9.6482 & 14.732 & 17.047 & 18.126 & 23.570 & 26.437 & 30.835 & 37.046 \\
\hline & FEM (shell) & 1.2675 & 4.4670 & 9.4575 & 14.210 & 16.525 & 17.494 & 22.646 & 25.456 & 29.524 & 35.410 \\
\hline & FEM (brick) & 1.2662 & 4.4643 & 9.4611 & 14.226 & 16.553 & 17.525 & 22.702 & 25.549 & 29.631 & 35.600 \\
\hline & Liew et al. [30] & 1.2673 & 4.4670 & 9.4580 & 14.210 & 16.525 & 17.494 & 22.646 & 25.456 & 29.525 & 35.415 \\
\hline \multirow[t]{3}{*}{3} & Present & 1.0574 & 3.6112 & 7.3749 & 12.671 & 14.707 & 16.974 & 19.585 & 21.131 & 26.491 & 27.653 \\
\hline & FEM (shell) & 1.0517 & 3.5797 & 7.2615 & 12.367 & 14.197 & 16.362 & 18.980 & 20.346 & 25.421 & 26.639 \\
\hline & FEM (brick) & 1.0506 & 3.5771 & 7.2614 & 12.379 & 14.214 & 16.386 & 19.025 & 20.391 & 25.497 & 26.746 \\
\hline \multirow[t]{3}{*}{3.5} & Present & 0.90326 & 3.0086 & 5.9339 & 9.9803 & 14.489 & 15.275 & 16.393 & 19.475 & 21.467 & 23.731 \\
\hline & FEM (shell) & 0.89850 & 2.9855 & 5.8590 & 9.7898 & 13.990 & 14.862 & 15.822 & 18.754 & 20.793 & 22.816 \\
\hline & FEM (brick) & 0.89755 & 2.9830 & 5.8574 & 9.7949 & 14.005 & 14.885 & 15.846 & 18.790 & 20.852 & 22.876 \\
\hline \multirow[t]{3}{*}{4} & Present & 0.78828 & 2.5782 & 4.9517 & 8.1529 & 12.229 & 14.569 & 15.890 & 17.236 & 18.431 & 21.763 \\
\hline & FEM (shell) & 0.78420 & 2.5601 & 4.8982 & 8.0224 & 11.959 & 14.061 & 15.326 & 16.761 & 17.770 & 20.938 \\
\hline & FEM (brick) & 0.78335 & 2.5579 & 4.8960 & 8.0242 & 11.971 & 14.077 & 15.347 & 16.795 & 17.803 & 20.987 \\
\hline \multirow[t]{3}{*}{4.5} & Present & 0.69929 & 2.2558 & 4.2439 & 6.8571 & 10.172 & 14.059 & 14.648 & 15.638 & 17.606 & 18.878 \\
\hline & FEM (shell) & 0.69567 & 2.2410 & 4.2034 & 6.7626 & 9.9815 & 13.675 & 14.182 & 15.091 & 16.970 & 18.344 \\
\hline & FEM (brick) & 0.69494 & 2.2389 & 4.2010 & 6.7624 & 9.9880 & 13.691 & 14.201 & 15.112 & 16.999 & 18.388 \\
\hline \multirow[t]{3}{*}{5} & Present & 0.62838 & 2.0053 & 3.7114 & 5.9004 & 8.6514 & 11.961 & 14.501 & 15.348 & 15.921 & 17.076 \\
\hline & FEM (shell) & 0.62517 & 1.9927 & 3.6793 & 5.8288 & 8.5106 & 11.711 & 13.995 & 14.814 & 15.507 & 16.471 \\
\hline & FEM (brick) & 0.62449 & 1.9908 & 3.6769 & 5.8275 & 8.5134 & 11.723 & 14.011 & 14.834 & 15.535 & 16.497 \\
\hline
\end{tabular}



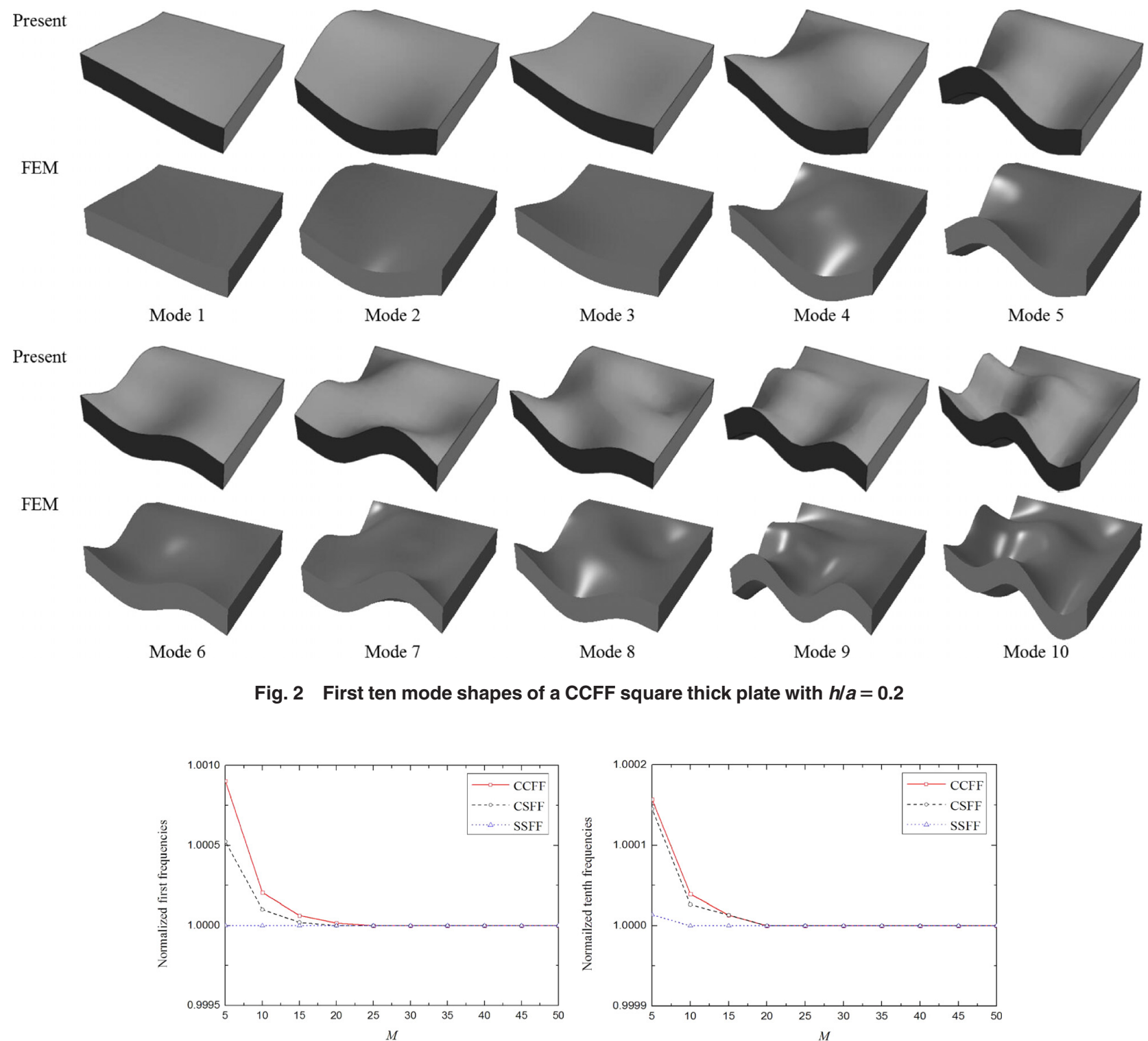

Mode 3

Mode 4

Mode 5
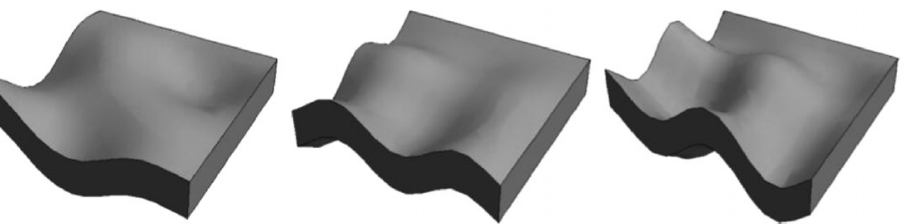

Mode 6
Mode 8

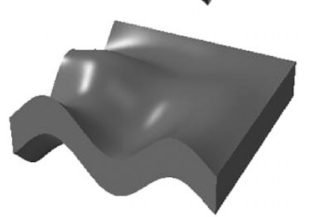

Mode 9

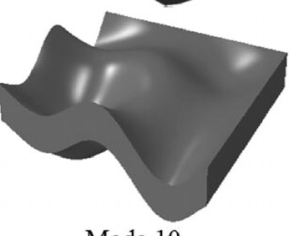

Mode 10

Fig. 2 First ten mode shapes of a CCFF square thick plate with $h / a=0.2$

Fig. 3 Convergence of the first and tenth normalized frequencies for CCFF, CSFF, and SSFF square plates with $h / a=0.2$

setting both $F_{m}=0$ and $H_{n}=0$, we obtain the solution of a SSFF plate.

\section{Comprehensive Numerical and Graphic Results}

To provide comprehensive natural frequency and mode shape solutions, we present many numerical and graphic results. In Tables $1-3$, we tabulate the first ten natural frequencies of CCFF plates with the aspect ratio $b / a$ ranging from 1 to 5 under an interval of 0.5 . The thickness-to-width ratio $h / a$ is set to be $0.05,0.1$, and 0.2 , respectively. Tables 4 and 5 correspond to CSFF and SSFF plates, respectively, with $h / a=0.2$ and $b / a$ being the same as those for Tables 1-3. The Poisson's ratio 0.3 is adopted throughout. All our analytic solutions are compared with their counterparts from the FEM via the ABAQUS software, in which both thick shell element S8R and linear brick element C3D8R are adopted [29]. Some numerical solutions by the Rayleigh-Ritz method in Ref. [30] are also listed for comparison. Figure 2 illustrates the mode shapes of thick enough $(h / a=0.2)$ square CCFF plates, from both our analytic and finite element solutions. A total of 450 frequencies ( 90 per table) and ten mode shapes are presented for benchmark purpose, all of which are well validated by

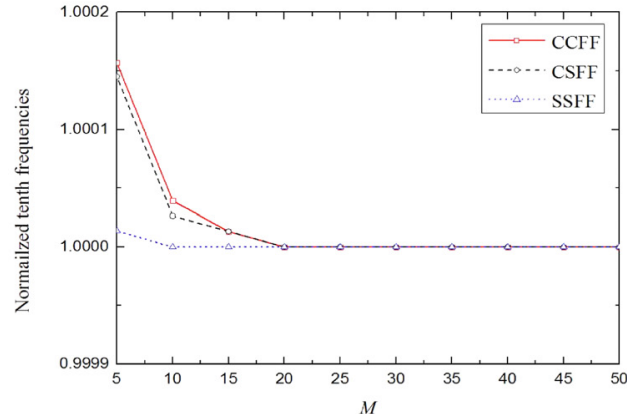

satisfactory agreement with the FEM and Ref. [30]. It should be noted that the minor differences between the present solutions and both the FEM and Ref. [30] increase for higher modes, which seems more obvious for thicker plates. This is probably due to the difference between the present plate theory neglecting the rotatory inertia and that incorporating the rotatory inertia (Ref. [30] and FEM with thick shell element) as well as three-dimensional elasticity (FEM with linear brick element).

The convergence study is carried out by plotting the first and tenth frequency solutions of the CCFF, CSFF, and SSFF square plates with $h / a=0.2$, normalized by their convergent values, versus the number of series terms (specified throughout as $M$ in this study), as shown in Fig. 3. It is verified that only 50 terms are adequate for achieving the accuracy of five significant figures for all current solutions; thus, $M=50$ is universally applied to yield the results.

\section{Conclusions}

New analytic free vibration solutions are obtained for the rectangular thick plates with a free corner based on the symplectic superposition method, including CCFF, CSFF, and SSFF plates. 
The governing equations are first transformed into the Hamiltonian system, where the effective mathematical techniques in the symplectic space such as the variable separation and symplectic eigen expansion are valid. A problem to be solved is treated as the sum of two fundamental problems that can be analytically solved with the above techniques. The equivalence between the original problem and the sum of fundamental problems leads to an equation to determine the frequencies. The mode shapes are then obtained without difficulty. The main advantage of the method is its ability to yield analytic solutions in a rational and rigorous way, without loss of fast convergence and accuracy. Comprehensive numerical and graphic results will play an important role in validating other new solution methods as the benchmarks.

\section{Funding Data}

- Young Elite Scientist Sponsorship Program by CAST (No. 2015QNRC003).

- National Basic Research Program of China (Grant No. 2014CB046506).

- National Natural Science Foundation of China (Grant Nos. 11302038, 11572323, and 11772331).

- Strategic Priority Research Program of the Chinese Academy of Sciences (No. XDB22040501).

- State Key Laboratory of Structural Analysis for Industrial Equipment, Dalian University of Technology (No. GZ1603).

- State Key Laboratory of Digital Manufacturing Equipment and Technology, Huazhong University of Science and Technology (No. DMETKF2017008).

\section{References}

[1] Leissa, A. W., 1969, Vibration of Plates, Office of Technology Utilization, NASA, Washington, DC

[2] Reissner, E., 1945, "The Effect of Transverse Shear Deformation on the Bending of Elastic Plates," ASME J. Appl. Mech., 12(2), pp. A69-A77.

[3] Mindlin, R. D., 1951, "Influence of Rotatory Inertia and Shear on Flexura Motions of Isotropic, Elastic Plates," ASME J. Appl. Mech., 18(1), pp. 31-38.

[4] Reddy, J. N., 1984, "A Simple Higher-Order Theory for Laminated Composite Plates," ASME J. Appl. Mech., 51(4), pp. 745-752.

[5] Reddy, J. N., 1984, "A Refined Nonlinear-Theory of Plates With TransverseShear Deformation,” Int. J. Solids Struct., 20(9-10), pp. 881-896.

[6] Wu, K., and Zhu, W., 2018, "A New Global Spatial Discretization Method for Calculating Dynamic Responses of Two-Dimensional Continuous Systems With Application to a Rectangular Kirchhoff Plate," ASME J. Vib. Acoust., 140(1), p. 011002.

[7] Wang, C. Y., 2015, "Vibrations of Completely Free Rounded Rectangular Plates," ASME J. Vib. Acoust., 137(2), p. 024502.

[8] Leamy, M. J., 2016, "Semi-Exact Natural Frequencies for Kirchhoff-Love Plates Using Wave-Based Phase Closure," ASME J. Vib. Acoust., 138(2), p. 021008.

[9] Waksmanski, N., Pan, E., Yang, L.-Z., and Gao, Y., 2014, "Free Vibration of a Multilayered One-Dimensional Quasi-Crystal Plate," ASME J. Vib. Acoust., 136(4), p. 041019
[10] Lai, S. K., and Xiang, Y., 2012, "Buckling and Vibration of Elastically Restrained Standing Vertical Plates," ASME J. Vib. Acoust., 134(1), p. 014502.

[11] Malekzadeh, P., and Karami, G., 2004, "Vibration of Non-Uniform Thick Plates on Elastic Foundation by Differential Quadrature Method," Eng. Struct. 26(10), pp. 1473-1482.

[12] Cho, D. S., Kim, B. H., Kim, J. H., Vladimir, N., and Choi, T. M., 2015 "Forced Vibration Analysis of Arbitrarily Constrained Rectangular Plates and Stiffened Panels Using the Assumed Mode Method," Thin-Walled Struct., 90, pp. 182-190.

[13] Zhou, D., Cheung, Y. K., Au, F. T. K., and Lo, S. H., 2002, "ThreeDimensional Vibration Analysis of Thick Rectangular Plates Using Chebyshev Polynomial and Ritz Method,” Int. J. Solids Struct., 39(26), pp. 6339-6353.

[14] Pradhan, K. K., and Chakraverty, S., 2015, "Transverse Vibration of Isotropic Thick Rectangular Plates Based on New Inverse Trigonometric Shear Deformation Theories," Int. J. Mech. Sci., 94-95, pp. 211-231.

[15] Ye, T, Jin, G, Su, Z, and Chen, Y, 2014, "A Modified Fourier Solution for Vibration Analysis of Moderately Thick Laminated Plates With General Boundary Restraints and Internal Line Supports," Int. J. Mech. Sci., 80, pp. $29-46$.

[16] Jin, G., Su, Z., Shi, S., Ye, T., and Gao, S., 2014, "Three-Dimensional Exact Solution for the Free Vibration of Arbitrarily Thick Functionally Graded Rectangular Plates With General Boundary Conditions," Compos. Struct., 108, pp. $565-577$.

[17] Zhang, H., Shi, D., and Wang, Q., 2017, “An Improved Fourier Series Solution for Free Vibration Analysis of the Moderately Thick Laminated Composite Rectangular Plate With Non-Uniform Boundary Conditions," Int. J. Mech. Sci., 121, pp. 1-20.

[18] Li, R., Wang, P., Tian, Y., Wang, B., and Li, G., 2015, "A Unified Analytic Solution Approach to Static Bending and Free Vibration Problems of Rectangular Thin Plates," Sci. Rep., 5, p. 17054.

[19] Li, R., Tian, Y., Wang, P., Shi, Y., and Wang, B., 2016, "New Analytic Free Vibration Solutions of Rectangular Thin Plates Resting on Multiple Point Supports," Int. J. Mech. Sci., 110, pp. 53-61.

[20] Wang, B., Li, P., and Li, R., 2016, "Symplectic Superposition Method for New Analytic Buckling Solutions of Rectangular Thin Plates," Int. J. Mech. Sci., 119, pp. 432-441.

[21] Xing, Y., and Liu, B., 2009, "Characteristic Equations and Closed-Form Solutions for Free Vibrations of Rectangular Mindlin Plates," Acta Mech. Solida Sin., 22(2), pp. 125-136.

[22] Xing, Y., and Liu, B., 2009, "Closed Form Solutions for Free Vibrations of Rectangular Mindlin Plates,” Acta Mech. Sin., 25, pp. 689-698.

[23] Li, R., Wang, P., Xue, R., and Guo, X., 2017, "New Analytic Solutions for Free Vibration of Rectangular Thick Plates With an Edge Free," Int. J. Mech. Sci., 131-132, pp. 179-190.

[24] Yao, W., Zhong, W., and Lim, C. W., 2009, Symplectic Elasticity, World Scientific, Singapore.

[25] Lim, C. W., and Xu, X. S., 2010, "Symplectic Elasticity: Theory and Applications," ASME Appl. Mech. Rev., 63(5), p. 050802.

[26] Lim, C. W., Lu, C. F., Xiang, Y., and Yao, W., 2009, “On New Symplectic Elasticity Approach for Exact Free Vibration Solutions of Rectangular Kirchhoff Plates," Int. J. Eng. Sci., 47(1), pp. 131-140.

[27] Lim, C. W., 2010, "Symplectic Elasticity Approach for Free Vibration of Rectangular Plates," J. Vib. Eng. Technol., 9(2), pp. 159-163.

[28] Li, R., Zhong, Y., and Li, M., 2013, "Analytic Bending Solutions of Free Rectangular Thin Plates Resting on Elastic Foundations by a New Symplectic Superposition Method,” Proc. R. Soc. A-Math. Phys. Eng. Sci., 469(2153), p. 20120681.

[29] ABAQUS, 2013, Analysis User's Guide V6.13, Dassault Systèmes, Pawtucket, RI.

[30] Liew, K. M., Xiang, Y., and Kitipornchai, S., 1993, "Transverse Vibration of Thick Rectangular Plates-1. Comprehensive Sets of Boundary Conditions," Comput. Struct., 49(1), pp. 1-29. 